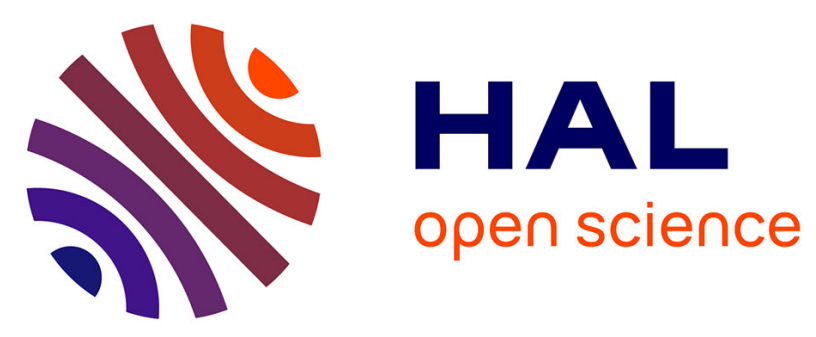

\title{
Genome-wide association studies for osteochondrosis in French trotter horses
}

Simon S. Teyssèdre, M.C. M. Dupuis, Gerard G. Guérin, Laurent L. Schibler, Jean-Marie Denoix, Jean Michel J. M. Elsen, Anne Ricard

\section{To cite this version:}

Simon S. Teyssèdre, M.C. M. Dupuis, Gerard G. Guérin, Laurent L. Schibler, Jean-Marie Denoix, et al. Genome-wide association studies for osteochondrosis in French trotter horses. Journal of Animal Science, 2012, 90 (1), pp.45-53. 10.2527/jas.2011-4031 . hal-01000230

\section{HAL Id: hal-01000230 \\ https://hal.science/hal-01000230}

Submitted on 29 May 2020

HAL is a multi-disciplinary open access archive for the deposit and dissemination of scientific research documents, whether they are published or not. The documents may come from teaching and research institutions in France or abroad, or from public or private research centers.
L'archive ouverte pluridisciplinaire HAL, est destinée au dépôt et à la diffusion de documents scientifiques de niveau recherche, publiés ou non, émanant des établissements d'enseignement et de recherche français ou étrangers, des laboratoires publics ou privés. 


\section{JOURNAL OF ANIMAL SCIENCE}

The Premier Journal and Leading Source of New Knowledge and Perspective in Animal Science

Genome-wide association studies for osteochondrosis in French Trotter horses S. Teyssèdre, M. C. Dupuis, G. Guérin, L. Schibler, J. M. Denoix, J. M. Elsen and A. Ricard

J ANIM SCI 2012, 90:45-53.

doi: 10.2527/jas.2011-4031 originally published online August 12, 2011

The online version of this article, along with updated information and services, is located on the World Wide Web at:

http://www.journalofanimalscience.org/content/90/1/45

WWw.asas.org 


\title{
Genome-wide association studies for osteochondrosis in French Trotter horses ${ }^{1}$
}

\author{
S. Teyssèdre, ${ }^{* 2,3}$ M. C. Dupuis,$\dagger^{2}$ G. Guérin, $\ddagger$ L. Schibler $₫+$ J. M. Denoix,$\dagger$ \\ J. M. Elsen,* and A. Ricard $\ddagger$ \\ *Institut National de la Recherche Agronomique (INRA), UR 631, 31326 Castanet-Tolosan, France; \\ $\dagger$ †cole Nationale Vétérinaire d'Alfort, Centre d'Imagerie et de Recherche sur les Affections Locomotrices \\ Equines, 14430 Goustranville, France; and $\ddagger$ INRA, UMR 1313, 78352 Jouy-en-Josas, France
}

\begin{abstract}
A genome-wide association study for osteochondrosis (OC) in French Trotter horses was carried out to detect QTL using genotype data from the Illumina EquineSNP50 BeadChip assay. Analysis data came from 161 sire families of French Trotter horses with 525 progeny and family sizes ranging from 1 to 20. Genotypes were available for progeny $(\mathrm{n}=525)$ and sires with at least 2 progeny $(\mathrm{n}=98)$. Radiographic data were obtained from progeny using at least 10 views to reveal OC. All radiographic findings were described by at least 2 veterinary experts in equine orthopedics, and severity indices (scores) were assigned based on the size and location of the lesion. Traits used were a global score, the sum of all severity scores lesions (GM, quantitative measurement), and the presence or
\end{abstract}

absence of $\mathrm{OC}$ on the fetlock (FM), hock (HM), and other sites (other). Data were analyzed using 2 mixed models including fixed effects, polygenic effects, and SNP or haplotype cluster effects. By combining results with both methods at moderate evidence of association threshold $P<5 \times 10^{-5}$, this genome-wide association study displayed 1 region for GM on the Equus caballus chromosome (ECA) 13, 2 for HM on ECA 3 and 14, and 1 for other on ECA 15 . One region on ECA 3 for $\mathrm{HM}$ represented the most significant hit $(P=3 \times$ $\left.10^{-6}\right)$. By comparing QTL between traits at a decreased threshold $\left(P<5 \times 10^{-4}\right)$, the 4 QTL detected for GM were associated to a QTL detected for FM or HM but never both. Another interesting result was that no QTL were found in common between HM and FM.

Key words: equine, French Trotter horse, genome-wide association, osteochondrosis, quantitative trait loci

(0)2012 American Society of Animal Science. All rights reserved.

J. Anim. Sci. 2012. 90:45-53 doi:10.2527/jas.2011-4031

\section{INTRODUCTION}

Osteochondral lesions are commonly observed in young horses and may be responsible for reduced performance in racing. The most frequent lesions, grouped under the generic name of osteochondrosis (OC), are osteochondritis dissecans lesions and bone cysts (Trotter and McIlwraith, 1981; Jeffcott and Henson, 1998). Fetlock, hock, and stifle joints are mainly affected. Osteochondrosis manifestations appear to be of multifactorial origin and many factors including genetic predisposition (Jeffcott, 1991; Philipsson et al., 1993;

\footnotetext{
${ }^{1}$ The GENEQUIN program was supported by the French National Research Agency (ANR, Paris), the Fonds Eperon (Paris, France), the French Institute for the Horse and Equitation (IFCE, Saumur), and the Basse-Normandie regional council (Caen, France).

${ }^{2}$ These authors contributed equally to this article.

${ }^{3}$ Corresponding author: simon.teyssedre@toulouse.inra.fr

Received March 7, 2011.

Accepted August 4, 2011.
}

Stock and Distl, 2006a; van Weeren, 2006), nutrition, exercise, and other environmental effects seem to play a role in its pathogenesis. However, the etiology and physiopathology of OC are not fully understood. Likewise, the molecular mechanisms involved in OC are still unknown.

The prevalence of OC in warmblood, coldblood, thoroughbred, and trotter horses ranges from 10 to $25 \%$ across different breeds (Grøndahl and Dolvik, 1993; Philipsson et al., 1993; Stock et al., 2005b; van Grevenhof et al., 2009). Heritability estimates for OC fall within the ranges $\mathrm{h}^{2}=0.17$ to 0.52 in trotters (Grøndahl and Dolvik, 1993; Philipsson et al., 1993) and $\mathrm{h}^{2}$ $=0$ to 0.37 in warmbloods (Schober et al., 2003; Stock et al., 2005a; Stock and Distl, 2006b).

Various whole-genome scans using microsatellite markers revealed QTL in Hanoverian warmblood breeds and South German coldbloods (Dierks et al., 2007; Wittwer et al., 2007, 2008, 2009). Those studies only shared 1 QTL on Equus caballus (ECA) 16. More recently, using the Illumina Bead-Chip EquineSNP50, 
2 genome-wide analyses were performed on Hanoverian warmblood (Lampe et al., 2010) and standardbred Norwegian horses (Lykkjen et al., 2010) and revealed several QTL in these 2 breeds.

The aim of this study was to carry out a genomewide association study using the Illumina BeadChip EquineSNP50 to identify QTL associated with OC in French Trotter horses (FT).

\section{MATERIALS AND METHODS}

The procedures involving animals consisted of radiography and collection of blood samples, which are routinely performed in veterinary practice and are considered noninvasive. All horses were sedated in accordance with clinical guidelines.

\section{Sample Characteristics}

Data were from the French GENEQUIN program, a study based on a population of FT. A total of $525 \mathrm{FT}$ were recorded. The sample came from 161 sire families with family sizes ranging from 1 to 20 with an average of 3.26 progeny per sire (63 sires had only 1 progeny). About $80 \%$ of the horses were less than 4 yr old at the time of examination (mean age $2.8 \pm 1.9$ ). About $45 \%$ of the sample horses were females and $55 \%$ males (with $23 \%$ geldings). Sixty-nine percent of the horses were in training when screened radiographically, and $40 \%$ had participated in at least 1 race. Genotypes were available for all progeny and for the 98 sires of 462 progeny (mean $4.7 \pm 3.6$ progeny per sire). More precisely, genotypes were obtained for all progeny and from sires with at least 2 progeny. Phenotypes, including all measurements, were available for all progeny only.

\section{Radiography}

Horses were recruited at CIRALE, the French Imaging and Research Center on Equine Locomotor Disorders, and a few veterinary clinics. Additional screening was performed on farms with increased OC prevalence in foals and yearlings to increase family sizes and collect DNA samples from dams when available. Information on clinical signs, medical history (to exclude cases having already undergone surgery), and horse origins were requested. Radiographies and all reports were examined by at least 2 veterinary experts in equine orthopedics. The radiologic screening consisted of at least 10 views including the lateromedial projections of the frontlimb and hindlimb digits (foot, pastern, and fetlock joints), dorsopalmar projections of the carpi, and lateromedial projections of the hocks and stifles.

\section{Phenotypic Measurements}

All radiographic findings were described accurately and severity indices (scores) were assigned based on the size and location of the lesion, as well as associated bone remodeling (Denoix et al., 2000). These scores were calculated as the power 2 of 4 severity degrees $(0$ to 3 ): the 0 degree represented a very small size lesion and corresponded to a score of $1\left(2^{\circ}=1\right)$; the first degree represented a small size lesion and corresponded to a score of $2\left(2^{1}=2\right)$; the second degree represented a medium size lesion and corresponded to a score of 4 ; the third degree represented a consistent size lesion and corresponded to a score of 8 . The frequency of lesions of degree $0(1,2$, and 3 , respectively) was $25 \%$ $(55,19$, and $1 \%$, respectively). A global score, the sum of the severity scores for all lesions on all radiographic sites, was attributed to each individual: horses with a global score of 0 were considered healthy; horses with a global score $>2$ were considered affected; horses with a global score of 1 , or with a global score of 0 or 2 but displaying unclear radiographic findings, were considered intermediate. To avoid erroneous results due to misclassification, intermediate horses were excluded from the analysis. Finally, based on global scores in our sample, 263 healthy horses and 262 affected horses were included in the analysis.

Different traits were used for data analysis. To obtain a phenotypic distribution as close as possible to a normal distribution, a global measurement (GM) was defined as the log-transformed of 1 plus global score $[\mathrm{GM}=\log (1+$ global score $)]$ and was analyzed as a continuous phenotype (Figure 1: mean $\pm \mathrm{SD}, 0.78 \pm$ $0.85)$. Site-specific analyses were also carried out after case-control studies based on the presence or absence of OC on the fetlock (FM), hock (HM), and other sites (other). The frequency of cases and controls are shown in Table 1. Pearson correlations between site-specific traits were rare (at the most 0.13), whereas GM was, by definition, highly correlated to the site-specific traits (Table 2).

\section{Markers and Genotype Quality Control}

Horse genotyping was performed using the Illumina Equine SNP50 BeadChip assay at Labogena, according to the manufacturer's standard procedures. This array includes 54,602 evenly distributed SNP throughout the genome. Markers were from all 31 equine autosomes and the $\mathrm{X}$ chromosome, and the average distance between $2 \mathrm{SNP}$ was $0.043 \pm 0.055 \mathrm{Mb}$. The $\mathrm{X}$ chromosome was not included in the analysis. All data were subject to quality control procedures. First, only samples showing a minimum call rate of $98 \%$ (percentage of SNP genotyped for an individual) were included in the study. Moreover, poor-quality markers were discarded based on 3 criteria: markers genotyped in less than $80 \%$ of the samples (call frequency $<80 \%$ ), having a minor allele frequency less than $5 \%$, or deviating from Hardy-Weinberg equilibrium in cases and controls $\left(P<10^{-8}\right)$ were rejected. Finally, 41,249 SNP were analyzed. 
Table 1. Frequency of osteochondrosis binary measurements ${ }^{1}$

\begin{tabular}{llccc}
\hline \hline Trait & Code & No. & Cases & Controls \\
\hline Fetlock & FM & 525 & 0.32 & 0.68 \\
Hock & HM & 525 & 0.23 & 0.77 \\
Other & Other & 525 & 0.11 & 0.89 \\
\hline
\end{tabular}

${ }^{1} \mathrm{FM}=$ fetlock measurement; $\mathrm{HM}=$ hock measurement; other $=$ osteochondrosis other than on the fetlock and the hock.

\section{Statistical Analyses}

Linkage Disequilibrium and Expected Power. Average linkage disequilibrium (LD) was computed every $0.02 \mathrm{Mb}$ across intervals of 0 to $1.6 \mathrm{Mb}$ using the $\mathrm{r}^{2}$ measure (Hill, 1974). The expected extent of LD assuming an effective population size of 150 and 1,000 individuals was also estimated using the method described by Tenesa et al. (2007). According to Ball (2005) and Luo (1998), the theoretical power of an association study performed on our sample size to detect a QTL in LD with a SNP was also estimated using R Package ldDesign software (R. Ball, http://cran.rproject.org/web/packages/ldDesign/index.html).

Single-SNP Analyses. To model the polygenic part and the SNP effect simultaneously, we performed the following mixed model:

$$
\mathbf{y}=1 \mu+\mathbf{X} \boldsymbol{\beta}+\mathbf{Z u}+\mathbf{e},
$$

with $\mathbf{y}$ is the vector of phenotypes, $\mu$ is the overall mean, $\boldsymbol{\beta}$ is the vector of the fixed effects with $\boldsymbol{\beta}^{\prime}=$ $\left(\boldsymbol{\beta}_{\mathrm{AGE}}, \boldsymbol{\beta}_{\mathrm{SNP}}\right), \mathbf{X}$ is the incidence matrix of $\boldsymbol{\beta}$ with the corresponding level of age at control and the genotypes of all individuals (coded 0,1 , and 2), $\mathbf{u}$ is the vector of random polygenic effects with $\mathbf{u} \sim N\left(0, \mathbf{A} \sigma_{u}^{2}\right), \mathbf{Z}$ is the incidence matrix for $\mathbf{u}$, and $\mathbf{e}$ is the vector of random residual effects with $\mathbf{e} \sim N\left(0, \mathbf{I} \sigma_{e}^{2}\right)$, where $\mathbf{I}$ is an identity matrix. The fixed effect "age at control," with 2 levels (individuals controlled at $2 \mathrm{yr}$ or less, and more than 2 yr), was included in this analysis for GM and HM. This effect was not significant for the other traits. The A matrix is the relationship matrix based on the available pedigree information that included 2,796 horses. Parameters of this model were estimated using ASReml (Gilmour et al., 2006) for each SNP on GM, HM, FM, and other. A Student's test of the null hypothesis (no

Table 2. Phenotypic correlation (Pearson) between traits $^{1}$

\begin{tabular}{lclll}
\hline \hline Trait & GM & FM & HM & Other \\
\hline GM & 1 & 0.64 & 0.63 & 0.43 \\
FM & - & 1 & 0.08 & 0.12 \\
HM & - & - & 1 & 0.13 \\
Other & - & - & - & 1
\end{tabular}

${ }^{1} \mathrm{GM}=$ global measurement of osteochondrosis; FM = fetlock measurement; HM = hock measurement; other $=$ osteochondrosis other than on the fetlock and the hock.

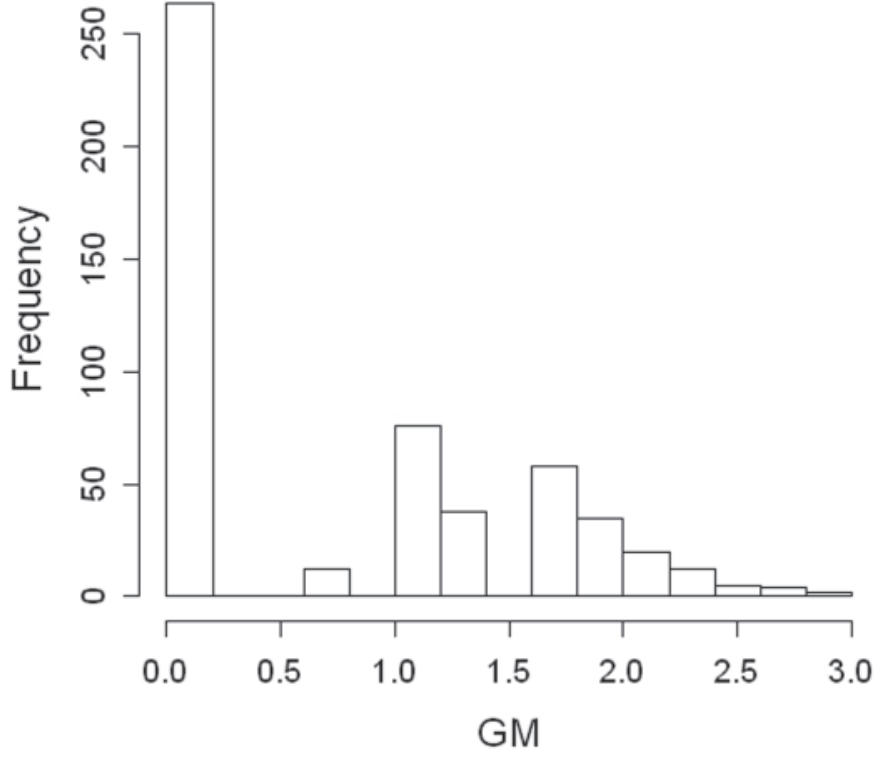

Figure 1. Distribution of the osteochondrosis global measurement (GM).

QTL, i.e., $\boldsymbol{\beta}_{\mathrm{SNP}}=0$ ) against the alternative (there is a QTL, i.e., $\boldsymbol{\beta}_{\mathrm{SNP}} \neq 0$ ) was performed for each SNP. We called this method SNPMixed. Heritabilities for all traits were also estimated with this model without the SNP effects.

Haplotype Analyses. We used the Druet et al. (2008) method, which combined linkage analysis and $\mathrm{LD}$ analysis. This method was based on the one described by Meuwissen et al. (2002) and derived from the original method proposed by Meuwissen and Goddard (2000). This is a variance component mapping method that includes information from LD between haplotypes and the transmission of haplotypes across generations. To provide complete information, the full procedure is described below. At first, we used the program DualPhase (Druet and Georges, 2010) to phase the haplotypes of all genotyped individuals. The sire haplotypes and the maternally inherited haplotypes of the sons were then considered as base haplotypes. When a sire of a progeny was not genotyped, base haplotypes were then progeny haplotypes. At each tested position, the following procedure was applied:

1. Probabilities of transmission, $\mathrm{p}_{\mathrm{ij}}$, were computed to determine which base haplotype corresponded to which paternally inherited haplotype. The rules applied when computing these probabilities using the closest informative bracket can be found in Table 1 in Pong-Wong et al. (2001). Linkage disequilibrium information was not taken into account at this step.

2. Identity-by-descent (IBD) probabilities $\left(\Phi_{\mathrm{p}}\right)$ were estimated among each pair of base haplotypes conditionally on the identity-by-state status of the neighboring markers using windows of 6 flanking markers (Meuwissen and Goddard, 2001). 
3. Base haplotypes were grouped with a clustering algorithm with Proc CLUST (SAS Inst. Inc., Cary, NC) using $\left(1-\Phi_{\mathrm{p}}\right)$ as a measure of distance. Base haplotypes were grouped if $\Phi_{\mathrm{p}}$ exceeded 0.6. Two additional rules were applied to assign haplotypes to clusters: i) when the 2 haplotypes of a sire were grouped in the same cluster, the paternally inherited haplotypes of all his sons were then grouped in this cluster, and ii) when the probability of transmission between a base haplotype and a haplotype was greater than 0.95 (it was grouped to the corresponding cluster).

4. The performances were modeled as follows:

$$
\mathbf{y}=\mathbf{X} \boldsymbol{\beta}+\mathbf{Z u}+\mathbf{Z}_{h} \mathbf{h}+\mathbf{e},
$$

where $\mathbf{y}$ is the vector of phenotypes, $\boldsymbol{\beta}$ is the vector of fixed effects including the overall mean and the effect "age at control," $\mathbf{X}$ is the incidence matrix of the fixed effects, $\mathbf{u}$ is the vector of random polygenic effects assumed to be normally distributed $\mathbf{u} \sim N\left(0, \mathbf{A} \sigma_{u}^{2}\right)$ with $\mathbf{A}$ the relationship matrix identical to the $\mathbf{A}$ matrix of SNPMixed, and $\mathbf{h}$ is a vector of random QTL effects corresponding to the haplotype clusters assumed to be normally distributed $\mathbf{h} \sim N\left(0, \mathbf{H} \sigma_{h}^{2}\right)$ with $\mathbf{H}$ the IBD matrix between haplotype clusters. The $\mathbf{Z}$ and $\mathbf{Z}_{h}$ are the design matrices relating phenotypes respectively to corresponding animal effects and haplotype clusters. The fixed effect "age at control" was only included in the model for GM and HM.

Maximum likelihood estimations of genetic parameters were obtained using an expectation-maximization REML. The REMLF90 software (Misztal et al., 2002) was modified by Druet et al. (2008) to incorporate relationship matrices among QTL allelic effects. The presence or absence of a QTL at a given position was tested with the likelihood-ratio test statistic:

$$
\lambda=-2 \ln \left[\frac{\mathrm{L}\left(\mathrm{H}_{0}\right)}{\mathrm{L}\left(\mathrm{H}_{1}\right)}\right],
$$

where $\mathrm{L}\left(\mathrm{H}_{0}\right)$ and $\mathrm{L}\left(\mathrm{H}_{1}\right)$ are the likelihood of the observations when parameters are equal to their REML estimation values under the polygenic model with no QTL fitted $\left(\mathrm{H}_{0}\right)$ and the general model with the QTL $\left(\mathrm{H}_{1}\right)$, respectively. The distribution of the test is not known but was previously shown to be close to onehalf of a 0 -df plus one-half of a 1-df $\chi^{2}$ distribution for a single position (Self and Liang, 1987). The $P$-values were computed using this distribution.

We applied this method to each SNP position on GM, HM, FM, and other. We called this method HaploIBD.

Criteria for Selecting Regions of Interest. Results obtained after a genome-wide association study with a dense marker map are made obscure by issues related to multiple testing and the high correlations between close markers due to LD. Multiple testing was controlled by applying stringent thresholds corresponding to $P$-values of $5 \times 10^{-4}, 5 \times 10^{-5}$, or $5 \times 10^{-6}$. The 41,249 tests performed were not independent because of the LD between SNP, and the most stringent threshold $\left(5 \times 10^{-6}\right)$ was chosen as an approximation of 10,000 independent tests corrected with Bonferroni (1936). The threshold at $5 \times 10^{-5}$ was used to provide moderate evidence of association (The Wellcome Trust Case Control Consortium, 2007), and the threshold at $5 \times 10^{-4}$ was used to describe and compare QTL between traits. Many of the markers with their $P$-values that exceeded the thresholds were actually correlated and could be grouped together. To avoid false detections resulting from the high correlation between markers in LD, the local score statistics proposed by Guedj et al. (2006) were used. The idea was to identify the chromosomal segments where the nonindependent tests were on average greater than a threshold due to the presence of a unique QTL. The greater the threshold, the smaller the segment size, but the greater the number of positive segments at the genome level. Because we wanted to take into account the error related to the detection of spurious QTL within a given chromosomal segment (resulting from genotype correlation at a QTL and markers in LD with this QTL), we chose a decreased threshold (corresponding to $20 \%$ of nonzero tests). This step gave a limited number of chromosomal regions where all positive signals could be explained by a unique QTL. Within each detected region with several correlated signals, we chose to keep only the greatest signal and identified it as the unique QTL.

\section{RESULTS}

\section{Heritability and $L D$}

Heritabilities were estimated at $0.32(\mathrm{SD} \pm 0.14)$ for GM, $0.27(\mathrm{SD} \pm 0.13)$ for $\mathrm{FM}, 0.45(\mathrm{SD} \pm 0.15)$ for $\mathrm{HM}$, and $0.13(\mathrm{SD} \pm 0.11$ ) for other. Figure 2 shows the estimated extent of $\mathrm{LD}\left(\mathrm{r}^{2}\right)$. The FT curve shows the estimated LD in our population of FT, whereas the other 2 curves (Ne150 and Ne1000) show the theoretical extent of LD in populations for which the effective sizes are, respectively, 150 and 1,000 individuals. The FT curve was closer to the Ne1000 curve for short distances and closer to the Ne150 curve for longer distances. This behavior suggests that the effective population size of FT decreased over generations. Using the Bead-Chip EquineSNP50, the mean distance between $2 \mathrm{SNP}$ is $0.043 \mathrm{Mb}$, which corresponds to an average LD of 0.25 (Figure 2). In this mean situation, at worst, a QTL is at a distance of about $0.02 \mathrm{Mb}$ from its 2 flanking SNP, which corresponds to a $0.35 \mathrm{LD}$. In this situation, considering bi-allelic markers and QTL, the detection power of our 525-individual design should reach 55, 78, and 91\% if QTL explain 3, 5, and 7\%, 


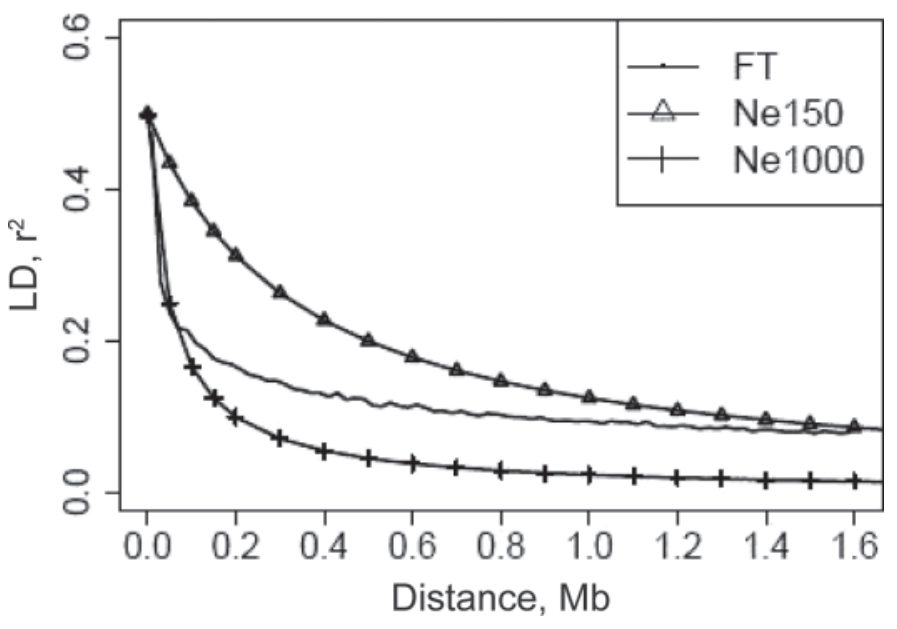

Figure 2. Extent of linkage disequilibrium $\left(L D ; r^{2}\right)$ in French Trotter horses (FT) assessed by using 525 samples from EquineSNP50 genotypes. The curve Ne150 (respectively Ne1000) represents the theoretical extent of $\mathrm{LD}$ in a population of effective size equal to 150 (respectively 1,000).

respectively, of the total phenotypic variance (Figure 3 ). Beyond this mean situation, set so that any present QTL could be detected, it should be noted that the LD between 2 SNP remains highly variable.

\section{QTL Detection}

Quantile-Quantile Plots. The quantile-quantile plots (QQPlots) informed us about the validity of the obtained $P$-values and the presence or absence of a population structure that might not have been taken into account in our models. Figure 4 shows the QQPlots obtained with SNPMixed and HaploIBD methods for GM, FM, HM, and other. Only the HaploIBD test on FM is slightly conservative; the others seem to follow the correct distribution.

Detected $Q T \boldsymbol{T}$. The results of QTL detection with rejection thresholds of $P<5 \times 10^{-4}, P<5 \times 10^{-5}$, and $P<5 \times 10^{-6}$ are presented in Table 3 . When the threshold was set at $P<5 \times 10^{-4}$, the SNPMixed method gave on average a larger number of QTL than HaploIBD (14.5 and 4.75, respectively). With this threshold, most of the QTL detected with HaploIBD were also detected with SNPMixed. We obtained 4 QTL common to both methods for GM, 2 for FM, and 4 for HM and other.

When the threshold was set at $P<5 \times 10^{-5}$, more QTL were again detected with SNPMixed than HaploIBD (4.25 and 1.25, respectively). Upon analysis of these QTL, all except for 1 detected on other were detected with both with the HaploIBD and SNPMixed methods. The QTL found in common for both methods for GM was located on ECA 13 (associated SNP were BIEC2-208655 and BIEC2-208753 with SNPMixed and HaploIBD, respectively), the 2 QTL found in common for HM were located on ECA 3 (BIEC2-808617 and BIEC2-808442) and 14 (BIEC2-265953 and BIEC2-265956), and the QTL found in common for other was located on ECA 15 (BIEC2-320636 and BIEC2-320532).

When the threshold was set at $P<5 \times 10^{-6}$, only 1 QTL, influencing HM, was detected on ECA 3 with HaploIBD. It was located between 100.39 and 107.92 $\mathrm{Mb}$ with a maximum at $105.05 \mathrm{Mb}$. At this position, the $P$-value was $-\log (P)=5.52$, and it was estimated that the QTL explained 7\% of the total phenotypic variance. With the SNPMixed method, the same QTL was not significant at $P<5 \times 10^{-6}$ but was significant at $P<5 \times 10^{-5}$. It was located with this method between 102.03 and $107.37 \mathrm{Mb}$ with a maximum at 105.88 $\mathrm{Mb}$. At this position, the $P$-value was $-\log (P)=4.94$ and the SNP effect accounted for 0.28 of the standard phenotypic variation. Manhattan plots obtained for GM, HM, FM, and other with HaploIBD are shown in Figures 5, 6, 7, and 8 .

Detected QTL Between Traits. The QTL that control distinct traits may be located closely, suggesting a pleiotropic effect. When the threshold was set at $P<5 \times 10^{-4}, 2$ of the 4 QTL detected for GM with both methods (SNPMixed and HaploIBD) were close to QTL detected for FM (on ECA 13 and 15), and the 2 others were close to QTL detected for HM (on ECA 3 and 14; Table 4). With this threshold, no QTL regions were shared by FM, HM, and other.

\section{DISCUSSION}

Animal populations are often composed of related individuals, and this is the case in our sample of FT. Association studies are sensitive to such population structures, and it is important to take them into account in the models. The 2 association methods, SNPMixed and

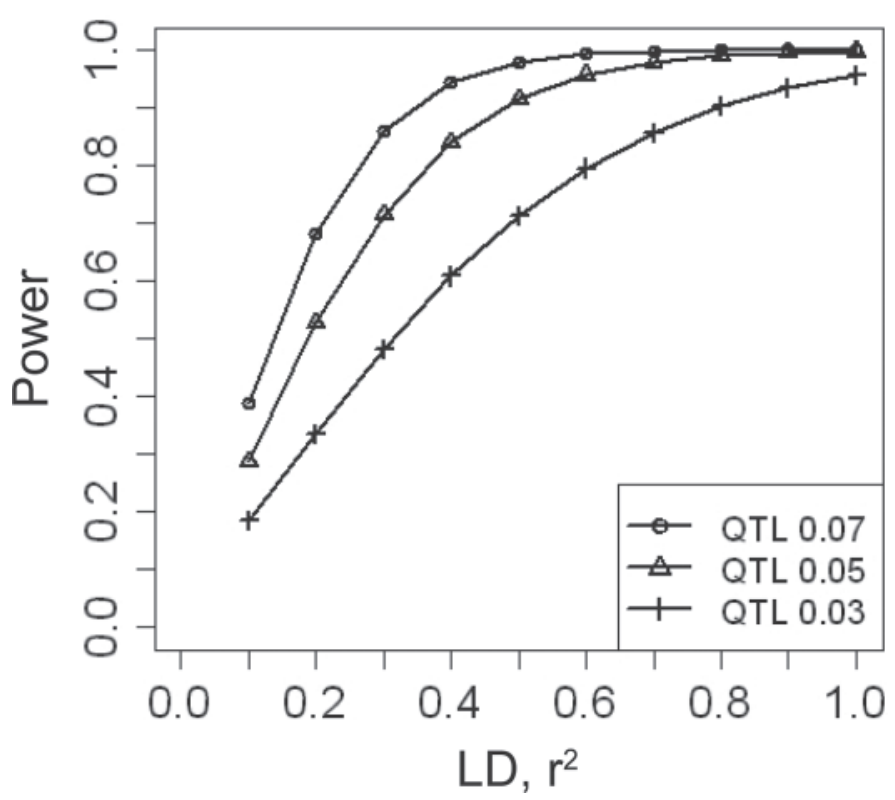

Figure 3. Theoretical power of association analysis in function of linkage disequilibrium (LD) between a bi-allelic marker and a QTL, explaining 3,5 , or $7 \%$ of the total phenotypic variance in a population size of 525 individuals. 
Table 3. Number of detected QTL at different thresholds

\begin{tabular}{llcccc}
\hline \hline Method $^{1}$ & Trait $^{2}$ & $P<5 \times 10^{-4}$ & $P<5 \times 10^{-5}$ & $P<5 \times 10^{-6}$ & $\operatorname{Max}^{3}$ \\
\hline SNPMixed & GM & 12 & 3 & 0 & 4.92 \\
& FM & 13 & 3 & 0 & 4.63 \\
& HM & 15 & 5 & 0 & 5.11 \\
HaploIBD & Other & 18 & 6 & 0 & 5.02 \\
& GM & 5 & 1 & 0 & 4.46 \\
& FM & 3 & 0 & 0 & 3.83 \\
& HM & 5 & 2 & 0 & 5.52 \\
Combined & Other & 6 & 2 & - & - \\
& GM & 4 & 1 & - & - \\
& FM & 2 & - & - & - \\
& HM & 4 & 2 & - & - \\
\hline
\end{tabular}

${ }^{1}$ SNPMixed, SNP mixed-model analyses; HaploIBD, haplotype mixed-model analyses; combined, QTL detected with both SNPMixed and HaploIBD methods.

${ }^{2} \mathrm{GM}=$ global measurement of osteochondrosis; $\mathrm{FM}=$ fetlock measurement; $\mathrm{HM}=$ hock measurement; other $=$ osteochondrosis other than on the fetlock and the hock.

${ }^{3} \operatorname{Max}=$ maximum $-\log _{10}(P)$.

HaploIBD, used in this study were robust to population structure (relationship structure), first by the use of a relationship matrix, and second with the use of linkage analysis for HaploIBD (Balding, 2006). This ensured a better control of false positives in the analysis. The QQPlots indicate that population structure had been accounted for by the analyses. Multiple tests could also create many false positives. Indeed, around 40,000 individual tests were performed. The classical Bonferroni correction (Bonferroni, 1936) was too strict because it assumes independence between each test, a hypothesis not fulfilled in our case. Tests were correlated, especially when using the haplotypes for 6 markers position after position. This is the reason we considered the use of different thresholds that were less stringent than Bonferroni's correction. There is no consensus on the choice of an ideal method or concerning SNP vs. haplotype analysis. By using both of these approaches and
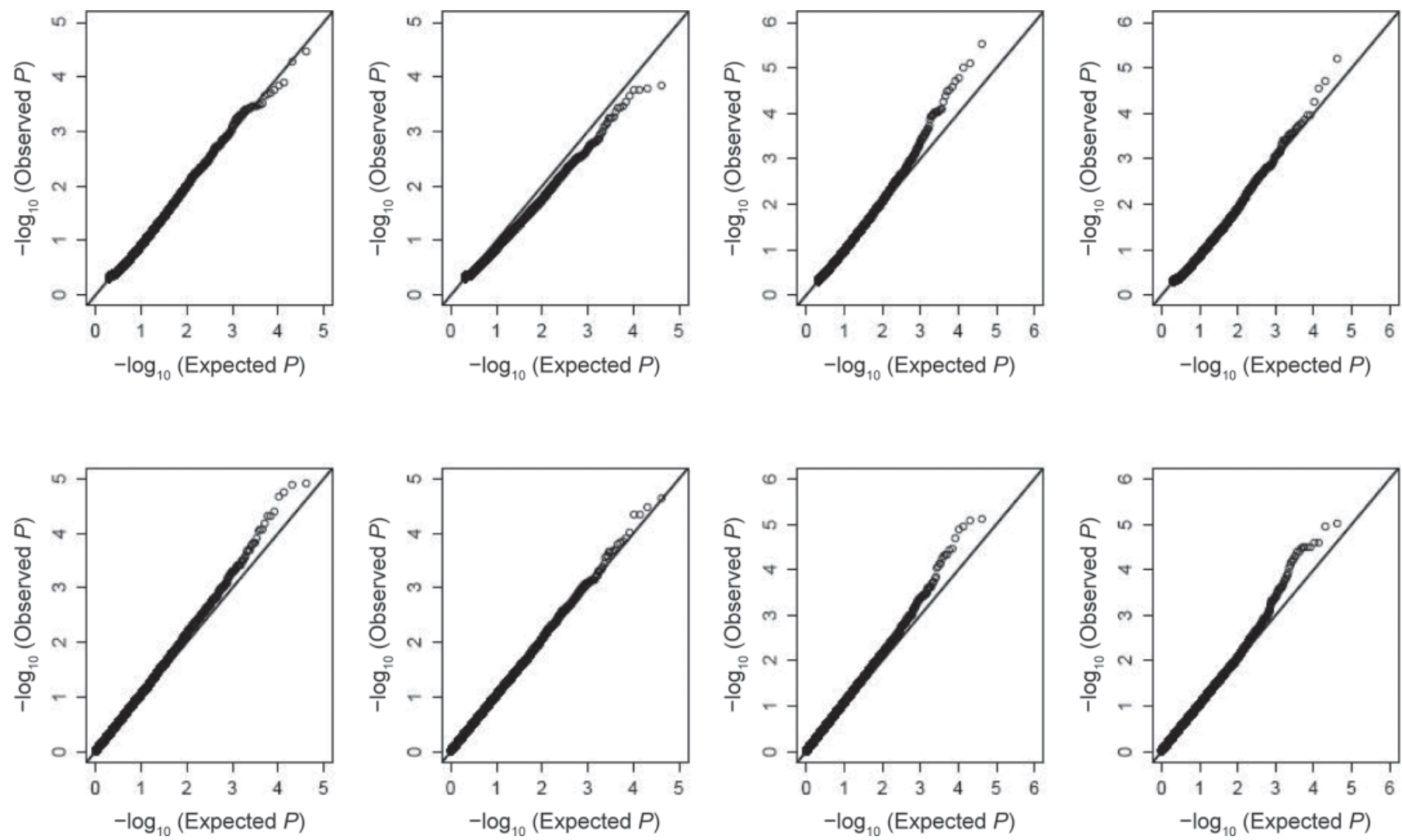

Figure 4. Quantile-quantile plots of $-\log _{10}$ of $P$-values resulting from haplotype mixed-model analyses (first line) and SNP mixed-model analyses (second line). Each column represents a measurement of osteochondrosis (OC): all sites, fetlock, hock, and other than fetlock and hock, respectively. Deviations from the slope line correspond to loci that deviate from the null hypothesis of no association. 


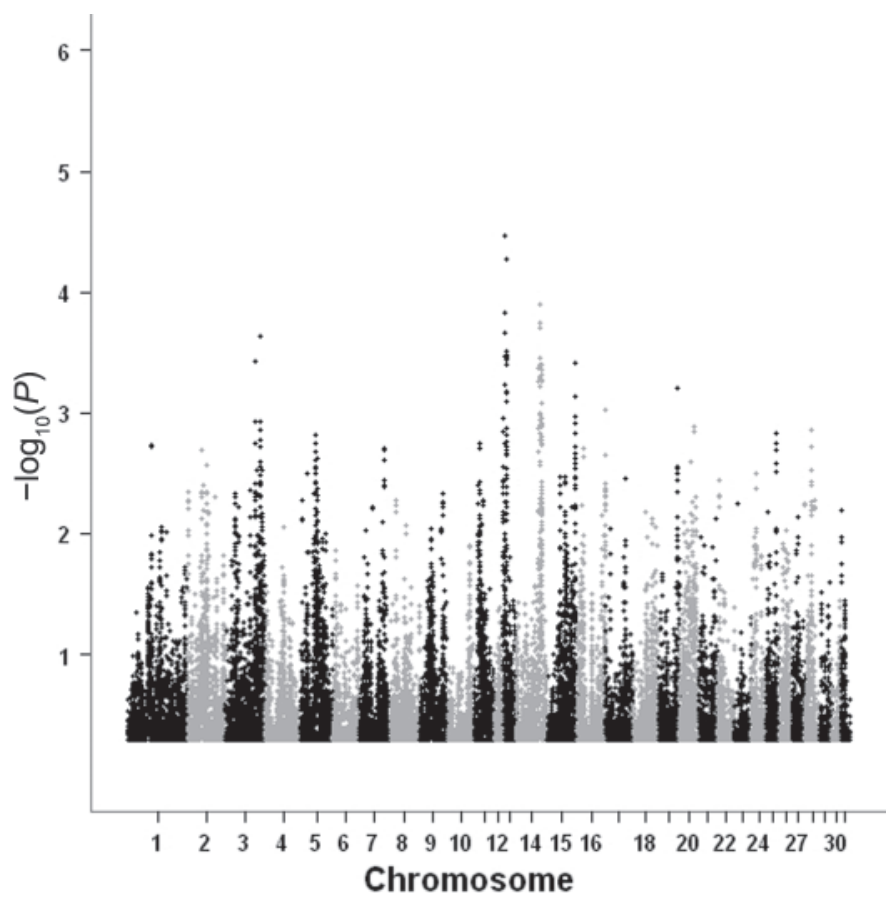

Figure 5. Manhattan plot of $-\log _{10}$ of $P$-values for the global measurement (GM) of osteochondrosis (scores based on all radiographic findings). The plot displays the haplotype mixed-model test results for GM at each SNP position on each chromosome.

combining their results, we expected to obtain a better accuracy and fewer false positives.

In this study, we detected 1 QTL on ECA 3 associated with $\mathrm{OC}$ of the hock at the $P<5 \times 10^{-6}$ sig-

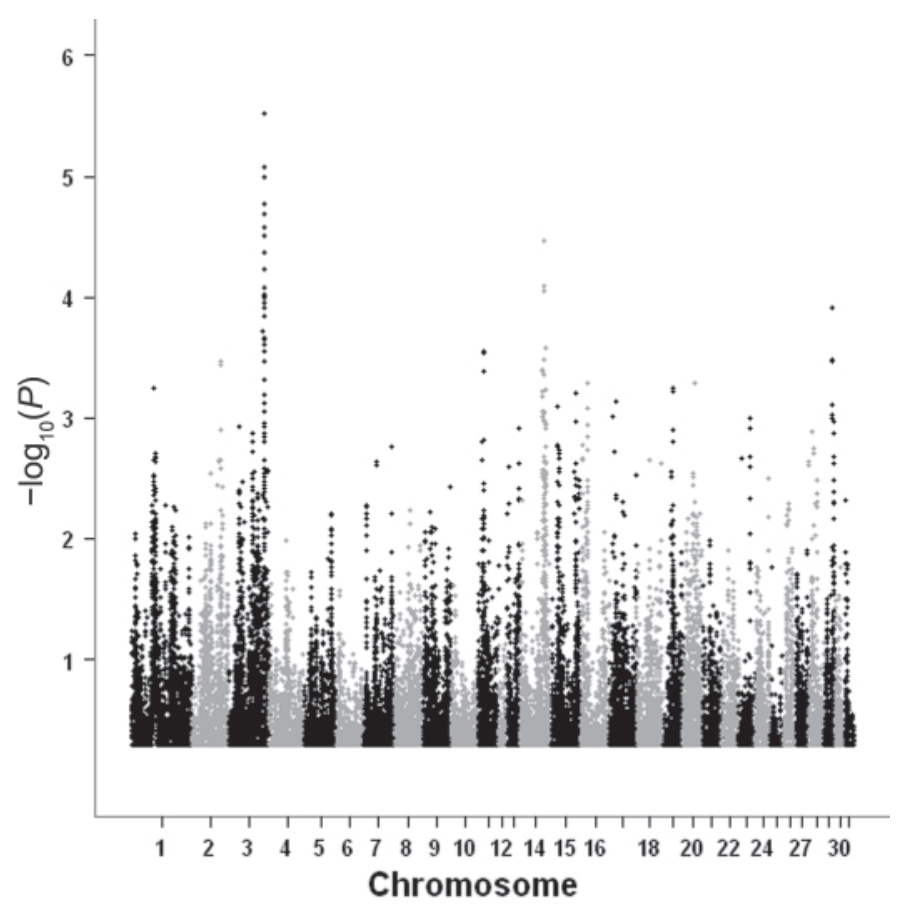

Figure 6. Manhattan plot of $-\log _{10}$ of $P$-values for the hock measurement (HM) of osteochondrosis (presence or absence of osteochondrosis on the hock). The plot displays the haplotype mixed-model test results for HM at each SNP position on each chromosome.

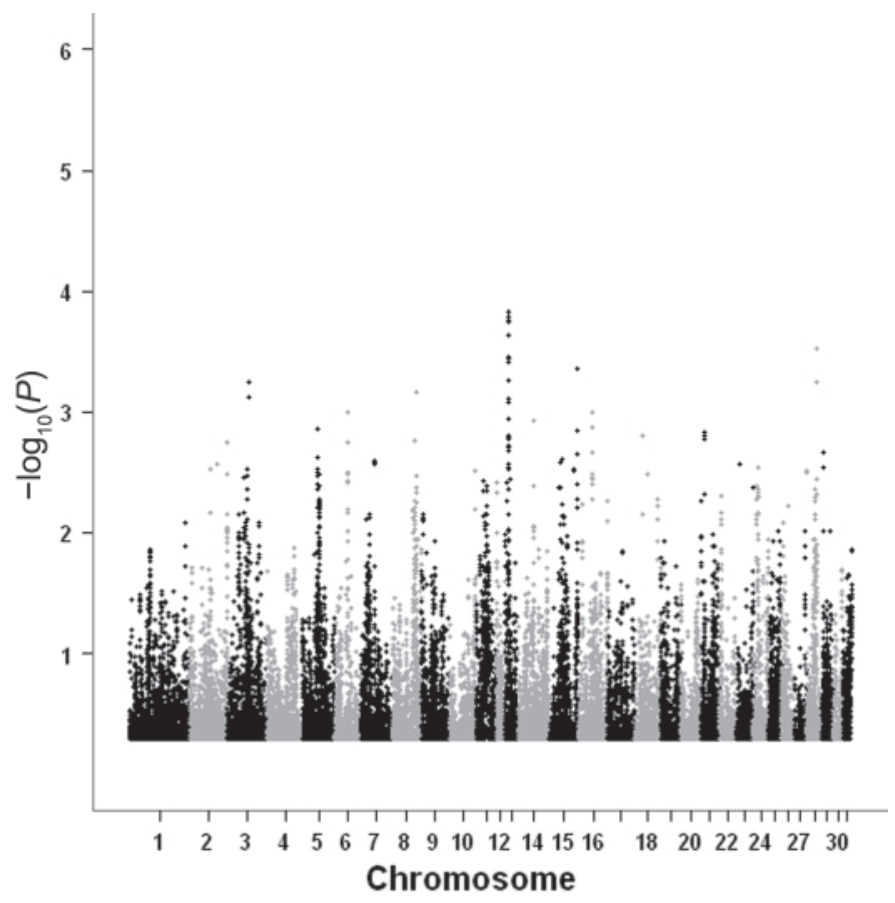

Figure 7. Manhattan plot of $-\log _{10}$ of $P$-values for the fetlock measurement (FM) of osteochondrosis (presence or absence of osteochondrosis on the fetlock). The plot displays the haplotype mixed-model test results for FM at each SNP position on each chromosome.

nificance level using a method that combined linkage analysis and LD and the use of haplotypes. Moreover, other regions were also associated with suggestive QTL for different traits but at decreased thresholds.

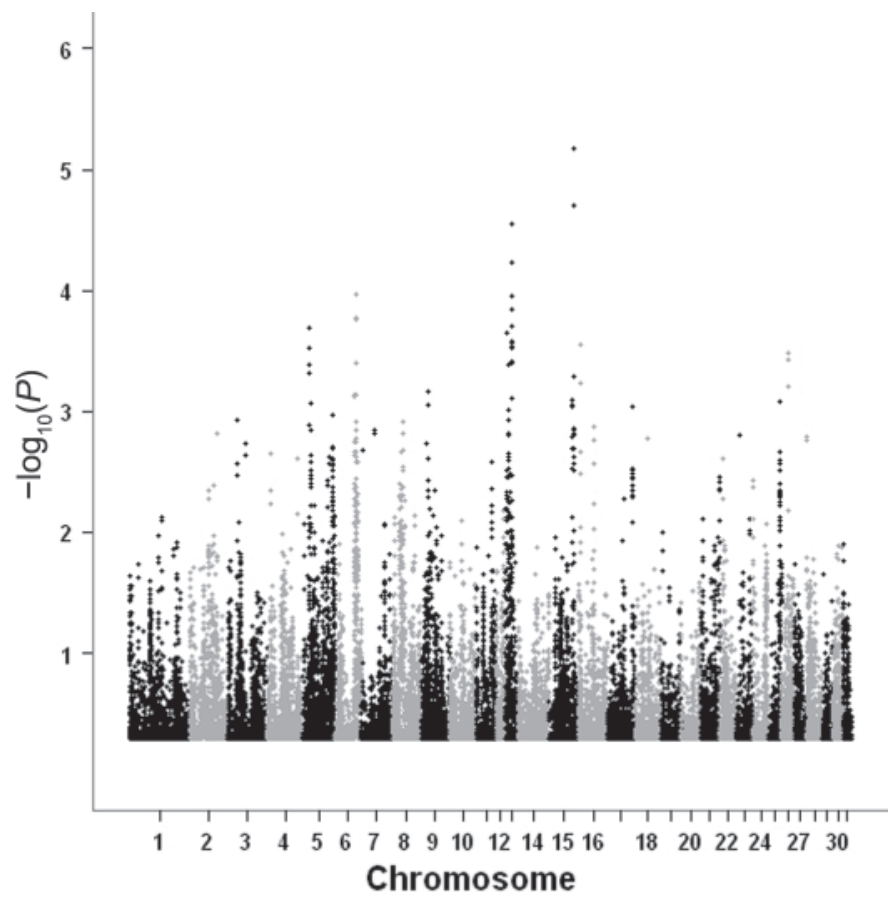

Figure 8. Manhattan plot of $-\log _{10}$ of $P$-values for the other measurement of osteochondrosis (presence or absence of osteochondrosis other than on the hock and the fetlock, other). The plot displays the haplotype mixed-model test results for the other measurement at each SNP position on each chromosome. 
Table 4. Quantitative trait loci between traits

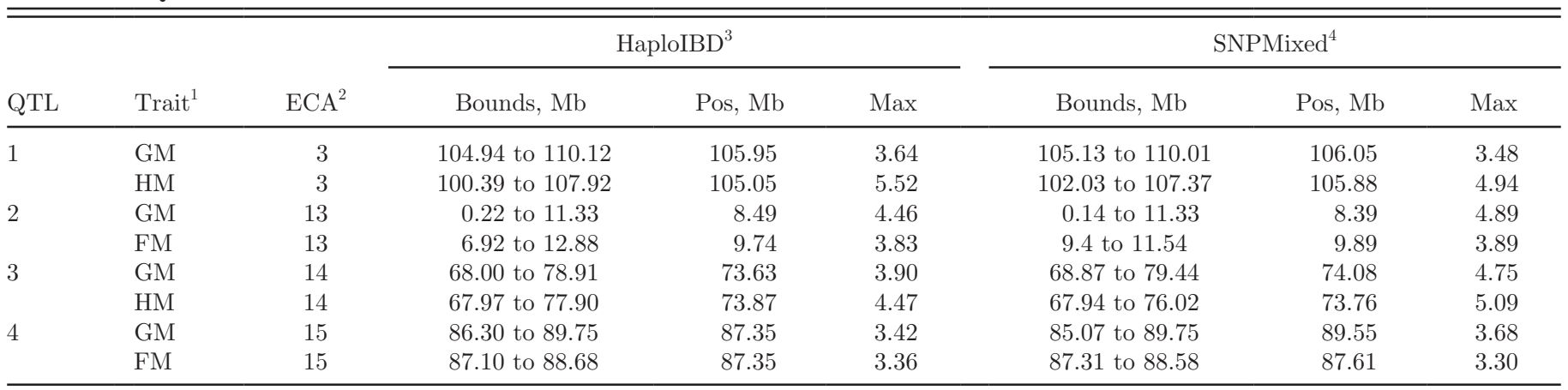

${ }^{1} \mathrm{GM}=$ global measurement of osteochondrosis; $\mathrm{FM}=$ fetlock measurement; HM = hock measurement.

${ }^{2} \mathrm{ECA}=$ Equus caballus chromosome.

${ }^{3}$ HaploIBD $=$ haplotype mixed-model analyses; Pos $=$ location of the QTL on the chromosome; $\mathrm{Mb}=\operatorname{megabase} ; \operatorname{Max}=\operatorname{maximum}$ of $-\log _{10}(P)$.

${ }^{4}$ SNPMixed $=$ SNP mixed-model analyses.

No QTL were found in common between HM and FM, an observation consistent with the decreased genetic correlations between $\mathrm{OC}$ of the hock and fetlock (Grøndahl and Dolvik, 1993; Stock and Distl, 2006b). All QTL detected for the GM of OC with the threshold $P<5 \times 10^{-4}$ were associated to a QTL detected for HM or FM but never both. These QTL were located on ECA 3 and 14 for HM and on ECA 13 and 15 for FM. As we expected from its definition, GM combines FM and HM and thus reveals specific QTL. Consistent with the greater incidence of cases observed for the fetlock than for the hock, one could expect that the GM would be more indicative of $\mathrm{OC}$ on the fetlock. However, the average score per lesion was greater for the hock than for the fetlock (2.62 and 2.05 before the log transformation, respectively), thus reversing the tendency.

Other QTL detection studies for OC were conducted in different breeds. Dierks et al. (2007) worked on Hanoverian warmblood horses and Wittwer et al. (2008) on South German coldblood horses. Both studies were performed using microsatellite markers in samples of approximately 200 horses. Most of the QTL regions detected in these studies were different, and in fact only a QTL on ECA 16 was found to be significant for FM between these breeds. The recent availability of the Illumina Bead-Chip EquineSNP 50 has allowed for genome-wide studies of OC susceptibility. As a result, Lampe et al. (2010) have reanalyzed the data of Dierks et al. (2007) in the Hanoverian warmblood sample, and mainly evidenced different QTL from those previously found with microsatellite markers. More recently, Lykkjen et al. (2010) studied 162 Norwegian SB horses with SNP and detected 9 regions of interest. No QTL were found in common to the 2 previous studies. However, 2 QTL on ECA 3 and 13 were found both in our study and 1 of the 2 previous ones, but at genome distances of about $5 \mathrm{Mb}$. Our study was based on a sample of 525 $\mathrm{FT}$, a population that is 2 to 3 times larger than in the 2 other OC studies using SNP. As a result, the power of our design was certainly more efficient as to detecting small and medium QTL effects (variance of QTL
$<5 \%$ ). A possible explanation for the differences in the QTL identified in the various studies could be that OC at different anatomical sites is associated with different loci in different breeds. Other explanations could include sample size or the various phenotypic criteria applied when selecting cases.

In conclusion, a few QTL associated with OC at different anatomical sites were revealed in this study. We show that OC, a multifactorial disease, is influenced by several genes, and we did not find that the genetic determinism is identical for OC developed on the hock and fetlock.

Further studies will focus on the identification of candidate genes and screening for mutation in an attempt to clarify the molecular physiopathology of $\mathrm{OC}$ and develop efficient strategies for risk assessment. Meanwhile, markers could be used in a marker-assisted selection context to improve horse health and welfare.

\section{LITERATURE CITED}

Balding, D. J. 2006. A tutorial on statistical methods for population association studies. Nat. Rev. Genet. 7:781-791.

Ball, R. D. 2005. Experimental designs for reliable detection of linkage disequilibrium in unstructured random population association studies. Genetics 170:859-873.

Bonferroni, C. E. 1936. Teoria statistica delle classi e calcolo delle probabilita. Libreria Internazionale Seeber, Italy.

Denoix, J. M., J. P. Valette, P. Heiles, X. Ribot, and L. Tavernier. 2000. Radiographic survey of juvenile osteoarticular lesions in 3 year old French breed horses: General results on 1180 horses. Pratique vétérinaire équine 32:35-41.

Dierks, C., K. Löhring, V. Lampe, C. Wittwer, C. Drögemüller, and O. Distl. 2007. Genome-wide search for markers associated with osteochondrosis in Hanoverian warmblood horses. Mamm. Genome 18:739-747.

Druet, T., S. Fritz, M. Boussaha, S. Ben-Jemaa, F. Guillaume, D. Derbala, D. Zelenika, D. Lechner, C. Charon, and D. Boichard. 2008. Fine mapping of quantitative trait loci affecting female fertility in dairy cattle on bta03 using a dense single-nucleotide polymorphism map. Genetics 178:2227-2235.

Druet, T., and M. Georges. 2010. A hidden Markov model combining linkage and linkage disequilibrium information for haplotype reconstruction and quantitative trait locus fine mapping. Genetics 184:789-798. 
Gilmour, A. R., B. J. Gogel, B. R. Cullis, and R. Thompson. 2006. Asreml User Guide Release 2.0. VSN International Ltd., Hemel Hempstead, UK.

Grøndahl, A. M., and N. I. Dolvik. 1993. Heritability estimations of osteochondrosis in the tibiotarsal joint and of bony fragments in the palmar/plantar portion of the metacarpo-and metatarsophalangeal joints of horses. J. Am. Vet. Med. Assoc. 203:101-104.

Guedj, M., D. Robelin, M. Hoebeke, M. Lamarine, J. Wojcik, and G. Nuel. 2006. Detecting local high-scoring segments: A first-stage approach for genome-wide association studies. Stat. Appl. Genet. Mol. Biol. 5:e22.

Hill, W. G. 1974. Estimation of linkage disequilibrium in randomly mating populations. Heredity 33:229-239.

Jeffcott, L. B. 1991. Osteochondrosis in the horse - Searching for the key to pathogenesis. Equine Vet. J. 23:331-338.

Jeffcott, L. B., and F. M. D. Henson. 1998. Studies on growth cartilage in the horse and their application to aetiopathogenesis of dyschondroplasia (osteochondrosis). Vet. J. 156:177-192.

Lampe, V., K. Komm, P. Lichtner, T. Meitinger, and O. Distl. 2010. Genome wide association analysis for osteochondrosis in Hanoverian warmblood horses using a SNP assay. University of Veterinary Medicine, Hannover, Germany.

Luo, Z. W. 1998. Detecting linkage disequilibrium between a polymorphic marker locus and a trait locus in natural populations. Heredity 80:198-208.

Lykkjen, S., N. I. Dolvik, M. E. McCue, A. K. Rendahl, J. R. Mickelson, and K. H. Roed. 2010. Genome wide association analysis of osteochondrosis of the tibiotarsal joint in Norwegian Standardbred trotters. Anim. Genet. 41:111-120.

Meuwissen, T. H. E., and M. E. Goddard. 2000. Fine mapping of quantitative trait loci using linkage disequilibria with closely linked marker loci. Genetics 155:421-430.

Meuwissen, T. H. E., and M. E. Goddard. 2001. Prediction of identity by descent probabilities from marker-haplotypes. Genet. Sel. Evol. 33:605-634.

Meuwissen, T. H. E., A. Karlsen, S. Lien, I. Olsaker, and M. E. Goddard. 2002. Fine mapping of a quantitative trait locus for twinning rate using combined linkage and linkage disequilibrium mapping. Genetics 161:373-379.

Misztal, I., S. Tsuruta, T. Strabel, B. Auvray, T. Druet, and D. H. Lee. 2002. Blupf90 and related programs (bgf90). Proc. 7th World Congr. Genet. Appl. Livest. Prod., Montpellier, France, August, Session 28.

Philipsson, J., E. Andreasson, B. Sandgren, G. Dalin, and J. Carlsten. 1993. Osteochondrosis in the tarsocrural joint and osteochondral fragments in the fetlock joints in standardbred trotters. II. Heritability. Equine Vet. J. 25:38-41.

Pong-Wong, R., A. W. George, J. A. Woolliams, and C. S. Haley. 2001. A simple and rapid method for calculating identity-bydescent matrices using multiple markers. Genet. Sel. Evol. 33:453-471.

Schober, M., M. Coenen, O. Distl, B. Hertsch, L. Christmann, and E. Bruns. 2003. Estimation of genetic parameters of osteochon- drosis (OC) in Hanoverian warmblood foals. Page 389 in 54th Annu. Meet. Eur. Assoc. Anim. Prod., Rome, Italy, Aug. 31 to Sep. 3.

Self, S. G., and K. Y. Liang. 1987. Asymptotic properties of maximum likelihood estimators and likelihood ratio tests under nonstandard conditions. J. Am. Stat. Assoc. 82:605-610.

Stock, K. F., and O. Distl. 2006a. Genetic correlations between conformation traits and radiographic findings in the limbs of German warmblood riding horses. Genet. Sel. Evol. 38:657-671.

Stock, K. F., and O. Distl. 2006b. Genetic correlations between osseous fragments in fetlock and hock joints, deforming arthropathy in hock joints and pathologic changes in the navicular bones of warmblood riding horses. Livest. Sci. 105:35-43.

Stock, K. F., H. Hamann, and O. Distl. 2005a. Estimation of genetic parameters for the prevalence of osseous fragments in limb joints of Hanoverian warmblood horses. J. Anim. Breed. Genet. 122:271-280.

Stock, K. F., H. Hamann, and O. Distl. 2005b. Prevalence of osseous fragments in distal and proximal interphalangeal, metacarpo and metatarsophalangeal and tarsocrural joints of Hanoverian warmblood horses. J. Vet. Med. Series A 52:388-394.

Tenesa, A., P. Navarro, B. J. Hayes, D. L. Duffy, G. M. Clarke, M. E. Goddard, and P. M. Visscher. 2007. Recent human effective population size estimated from linkage disequilibrium. Genome Res. 17:520-526.

Trotter, G. W., and C. W. McIlwraith. 1981. Osteochondrosis in horses: Pathogenesis and clinical syndromes. Proc. Annu. Conv. Am. Assoc. Equine Pract. 27:141-160.

van Grevenhof, E. M., A. Schurink, B. J. Ducro, P. R. van Weeren, J. M. F. M. van Tartwijk, P. Bijma, and J. A. M. van Arendonk. 2009. Genetic parameters of various manifestations of osteochondrosis and their correlations between and within joints in Dutch warmblood horses. J. Anim. Sci. 87:1906-1912.

van Weeren, P. R. 2006. Etiology, diagnosis, and treatment of OC(D). Clin. Tech. Equine Pract. 5:248-258.

The Wellcome Trust Case Control Consortium. 2007. Genome-wide association study of 14,000 cases of seven common diseases and 3,000 shared controls. Nature 447:661-678.

Wittwer, C., C. Dierks, H. Hamann, and O. Distl. 2008. Associations between candidate gene markers at a quantitative trait locus on equine chromosome 4 responsible for osteochondrosis dissecans in fetlock joints of south German Coldblood horses. J. Hered. 99:125-129.

Wittwer, C., H. Hamann, and O. Distl. 2009. The candidate gene XIRP2 at a quantitative gene locus on equine chromosome 18 associated with osteochondrosis in fetlock and hock joints of south German Coldblood horses. J. Hered. 100:481-486.

Wittwer, C., K. Löhring, C. Drögemüller, H. Hamann, E. Rosenberger, and O. Distl. 2007. Mapping quantitative trait loci for osteochondrosis in fetlock and hock joints and palmar/plantar osseus fragments in fetlock joints of south German Coldblood horses. Anim. Genet. 38:350-357. 
References

Citations

This article cites 30 articles, 9 of which you can access for free at: http://www.journalofanimalscience.org/content/90/1/45\#BIBL

This article has been cited by 1 HighWire-hosted articles: http://www.journalofanimalscience.org/content/90/1/45\#otherarticles 